\title{
Researching Specialized Languages
}

\section{Vijay Bhatia, Purificación Sánchez Hernández, Pascual Pérez-Paredes (eds). \\ Amsterdam/Philadelphia: John Benjamins, 2011. 238 pages. ISBN: 978-90-272-0352-6.}

In the last few decades, the field of specialised languages (LSP) has attracted increasing attention not only as a discipline in itself but also as a multidisciplinary area which feeds from and serves other related disciplines. This collection of articles, edited by Vijay Bhatia, Purificación Sánchez Hernández and Pascual Pérez-Paredes, offers a varied and rich perspective on LSP with the insight of corpus analysis and discourse and genre analysis, among others. The volume represents a welcome and relevant contribution to the study of a wide range of topics within the domain of LSP, from micro-genres such as conference abstracts to the meta-analysis of the lexicogrammar of specialised discourses.

This edited volume is divided into two sections: the first part, entitled "Research based on corpora", includes six chapters which have in common the corpus-based approach they take in their respective studies. The second section, "Research based on meta-analysis and applications in LSP", is made up of five chapters which apply meta-analysis as their main research methodology and offer interesting applications in the area of specialised languages.

Chapter one, "The historical shift of scientific academic prose in English towards less explicit styles of expression: Writing without verbs", by DOUglas BiBer and Bethany Gray, offers a historical analysis of the grammatical devices used in research articles to mark explicitness and structural elaboration, in contrast with the patterns found in conversation. The study draws on a 3-million-word corpus of research papers from four disciplines: Medicine, Education, Psychology and History. Pedagogical implications for the teaching of academic reading and writing at the university level are also discussed.

The second chapter, "Heteroglossic (dis)engagement and the construal of the ideal readership: Dialogic spaces in academic texts", by CArmen PérezLLANTAdA Auría, examines the rhetorical functionality of specific lexico- 
grammatical patterns for the expression of inter-subjective stance. Drawing on three corpora of biomedical research articles written in English and Spanish, the author explores the occurrence of we-subject patterns, anticipatory it-patterns, inanimate subject patterns and passive constructions across the various communicative sections and, following White (2003), establishes a distinction between two modes of heteroglossic (dis)engagement with the audience.

In "Structure, content and functions of calls for conference abstracts", SARA GESUATO analyses the pragmatic functions and structural organisation of 100 calls for conference abstracts (CfCAs) in a variety of disciplines, namely Biology, Computing, History and Linguistics. She defines the CfCAs as "representative, directive and commissive texts" (page 47) which display sixteen different move types, with the main move, "Invitation", carrying the genre's principal communicative purpose. The supporting or auxiliary moves, on the other hand, fulfil informative and persuasive-promotional functions.

Chapter four, "Summarizing findings: An all-pervasive move in open access biomedical research articles involves rephrasing strategies", by MERCEDES JAIME-SisÓ, explores the way biomedical scientists deal with their professional reading and writing. The paper shows that the rhetorical structure of scientific articles is gradually changing to meet the needs of online readers, which accounts for the repetition of findings and the paraphrasing of statements in all the sections, especially in open access publications.

“The use of adverbial hedges in EAP students' oral performance: A crosslanguage analysis", by Pascual Pérez-Paredes, Purificación Sánchez Hernández and Pilar Aguado-Jiménez, offers an interview-based comparative study of the use of the hedges "almost", "maybe", "sort of" and "kind of" in the oral production of two groups of speakers: Spanish learners of EAP and British students, both at a university level. The results indicate shortcomings in the performance of the first group of learners due to lack of formal instruction in the use of hedging devices, while the higher frequency of use of adverbial hedges among native speakers points at a lower lexical density in spoken communication.

CARMen SANCHO Guinda writes the closing chapter of the first section, "Integrating approaches to visual data commentary: An exploratory case study". Here, the author argues for the status of data commentary as a 
"multi-skill and cross-disciplinary practice" (page 115) frequently used in ESP environments, and proposes her own model drawing on the taxonomy developed by Systemic Functional Linguistics (Halliday, 1987). The author also offers a case study on the use of data transfer and commentary by Aeronautical Engineering students with an intermediate level of proficiency in English.

The second part of the volume opens with JOHN FLOWERDEW's chapter "Some dichotomies in genre analysis for Languages for Specific Purposes", where he discusses four dichotomies for genre research, namely individual genres vs. genre networks, written vs. spoken genres, macro vs. micro levels of analysis, and move structure vs. lexico-grammar. The main purpose of the chapter is "to highlight areas where further theoretical reflection and empirical enquiry might enhance the theory and at the same time offer even more for application" (page 151).

"English for legal purposes and domain-specific cultural awareness: The 'continental paradox', definition, causes and evolution”, by SHAEDA IsANI, identifies the phenomenon known as the "continental paradox", which basically refers to the fact that French, German and Spanish law students know the American legal system better than their own. Drawing on imprinting and acquisition theories, Isani analyses the three-phase cognitive process which underlies the acquisition of the ersatz legal culture.

In "The Talking Cure: From Narrative to Academic Argument", GILLIAN DiANE LAZAR focuses on a somewhat neglected genre in the teaching of academic writing at the tertiary level: the narrative genre, especially of an oral nature. The author offers a description of the writing difficulties faced by British university students and argues that narrative discourse can be used as an effective classroom tool to introduce them into academic writing. The paper also suggests practical techniques to be used in the classroom, such as triangulation, draft-writing and annotation.

"UrgentiAS, a lexical database for medical students in clinical placements: Architecture, use and evaluation", by Kris Buyse, Eva SAVEr, An LAFFut and Herlinda Vekemans, presents an innovative online multilingual medical lexicon devised to help Dutch-speaking Belgian students acquire the vocabulary they need in specific communicative situations during their clinical placements in the areas of obstetrics and gynaecology, paediatrics, and internal medicine in hospitals abroad. In order to build this multilingual database, the authors compiled an open, synchronic and 
multilingual corpus using material from different monolingual corpora and varied text types.

The concluding chapter, "Using natural language patterns for the development of ontologies", by Elena Montiel-Ponsoda and Guadalupe Aguado DE CEA, explores lexical relations in the context of ontology development. More specifically, the authors focus on the so-called "lexico-syntactic patterns", combinatory units which follow a certain syntactic order, with the objective of creating a repository associated to the ontological structures expressed. This will prove especially useful to researchers working in the domains of terminology, translation and the teaching of specialised languages.

Overall, the articles included in this carefully edited collection are theoretically well-grounded, and undoubtedly contribute, each in its own way, to the development of LSP, opening up interesting lines for future research. However, the diversity of the topics unfortunately results in a certain lack of cohesion and unity of the book as a whole. Similarly, given the wide range of research interests addressed in the book, it is difficult to envisage one homogeneous type of audience. Despite these shortcomings, the volume represents a most valuable contribution to the field of LSP and is highly recommendable to teachers and researchers whose interests lie in specialised languages, academic and professional writing, terminology and lexicography.

[Review received 24 September 2013]

[Revised review accepted 15 November 2013]

Reviewed by Maria-Lluïsa Gea-Valor

Universitat Jaume I (Spain)

gea@uji.es

\section{References}

Halliday, M.A.K. (1987). An Introduction to Functional Grammar. London: Edward Arnold.

White, P.R.R. (2003). "Beyond modality and hedging: A dialogic view of the language of intersubjective stance". Text 23: 259-284. 